\title{
A patient with haemoptysis, rapidly changing pulmonary infiltrates and left flank pain
}

\section{Case history}

A 28 year old male patient sought medical advice because of a 2 week history of haemoptysis. He began to suffer from vague right chest pain 1 month earlier, followed by intermittent fever, progressive dyspnoea and cough with blood clots in the sputum. Occasional left upper quadrant and left flank pain were also noted. He lost $6 \mathrm{~kg}$ in weight during this period. Pneumonia was suspected from the initial chest radiograph (fig. 1a) at a local hospital, and antibiotics were prescribed for 5 days but without significant benefit. The patient was referred to this hospital for a second opinion.

The past medical history was noncontributory. Physical examination revealed a well-developed man in mild respiratory distress. The body temperature was $37^{\circ} \mathrm{C}$, blood pressure was $130 / 70 \mathrm{mmHg}$, pulse 100 beats $\cdot \mathrm{min}^{-1}$, and respiratory rate 30 breaths. $\mathrm{min}^{-1}$. Breathing sounds were diminished in bilateral lower lung fields, with some

a)

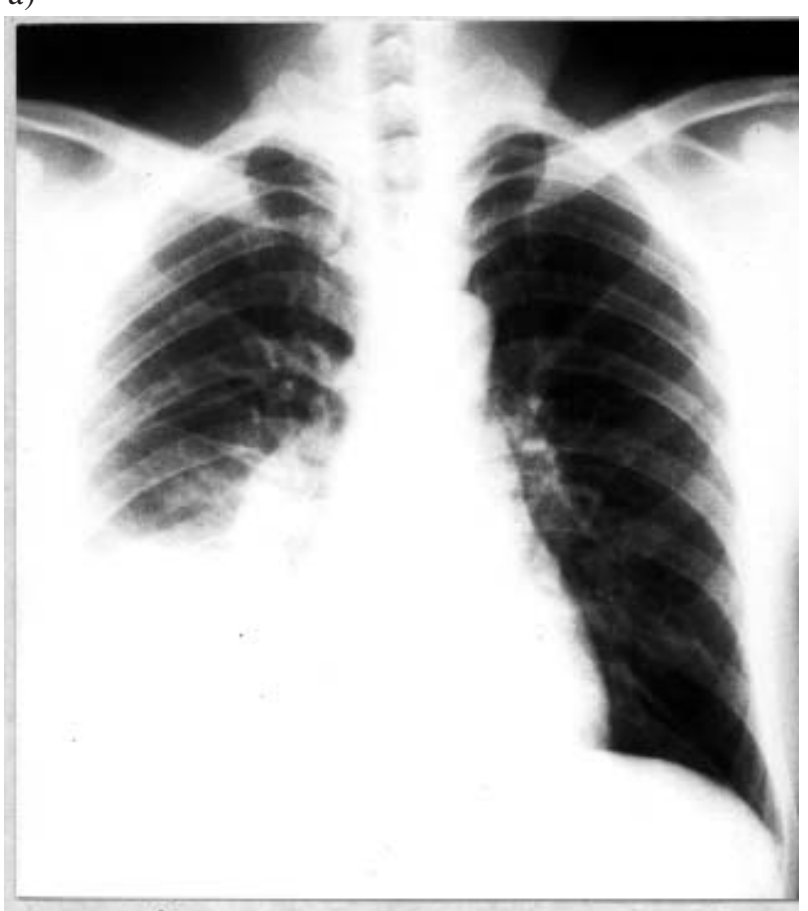

crackles. Heart sounds were regular and without murmurs. There was tenderness of the left costovertebral angle and mild swelling of the right thigh. No skin rash was detected, with the exception of some ecchymosis at the right inguinal area. Neither digital clubbing nor splinter haemorrhage was found. A complete blood count revealed: white blood cells (WBC) $24.4 \times 10^{9}$ cells $\cdot \mathrm{L}^{-1}$; haemoglobin $(\mathrm{Hb}) 110 \mathrm{~g} \cdot \mathrm{L}^{-1}$; and $86 \times 10^{9}$ platelets $\cdot \mathrm{L}^{-1}$. Determination of arterial blood gas values in room air showed: a $\mathrm{pH}$ of 7.48 ; arterial carbon dioxide tension $\left(P \mathrm{a}, \mathrm{CO}_{2}\right)$ of $4.8 \mathrm{kPa}(36 \mathrm{mmHg})$; and arterial oxygen tension $\left(\mathrm{Pa}, \mathrm{O}_{2}\right)$ of $9.4 \mathrm{kPa}(71 \mathrm{mmHg})$. Prothrombin time and partial prothrombin time were both prolonged. Sputum was negative for acid-fast bacilli (AFB), fungi and malignant cells. Urinalysis was normal.

Imaging studies, including a follow-up chest radiograph 5 days later (fig. 1b), the sequential contrastenhanced computed tomographic (CT) scans at the level of the aorticopulmonary window (fig. 2a), of the lower thorax (fig. 2b), and of both kidneys (fig. 3), are shown.

b)

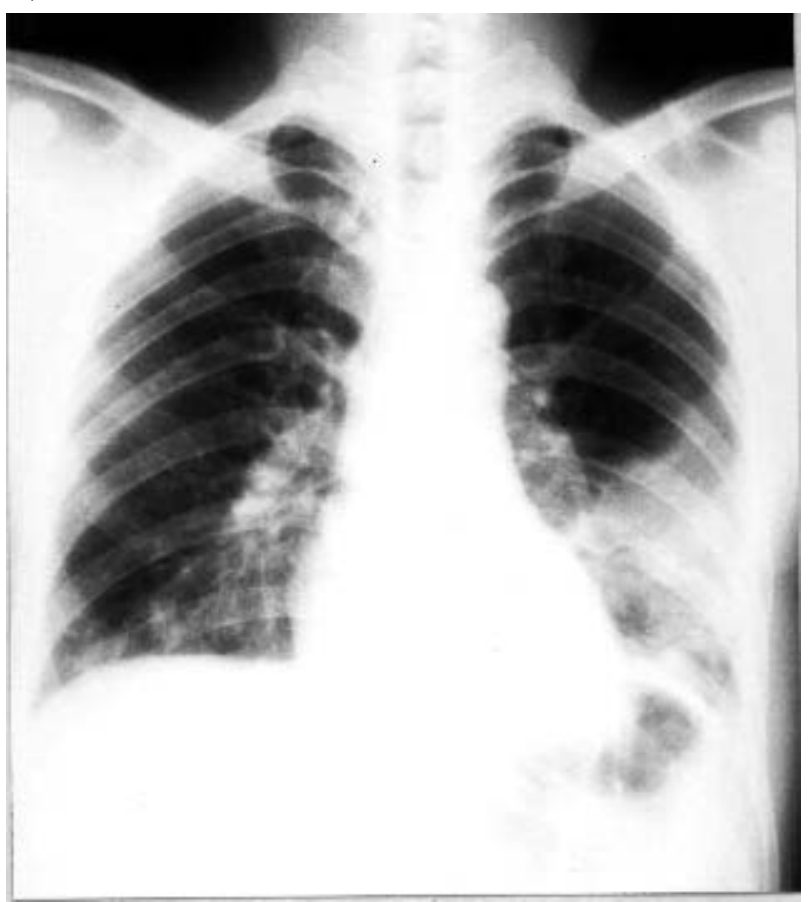

Fig. 1. - a) The initial posteroanterior chest radiograph; b) follow-up chest radiograph 5 days later.

Correspondence: P-C Yang, Dept of Internal Medicine, National Taiwan University Hospital, No. 7, Chung-Shan South Road, Taipei, Taiwan. 
a)
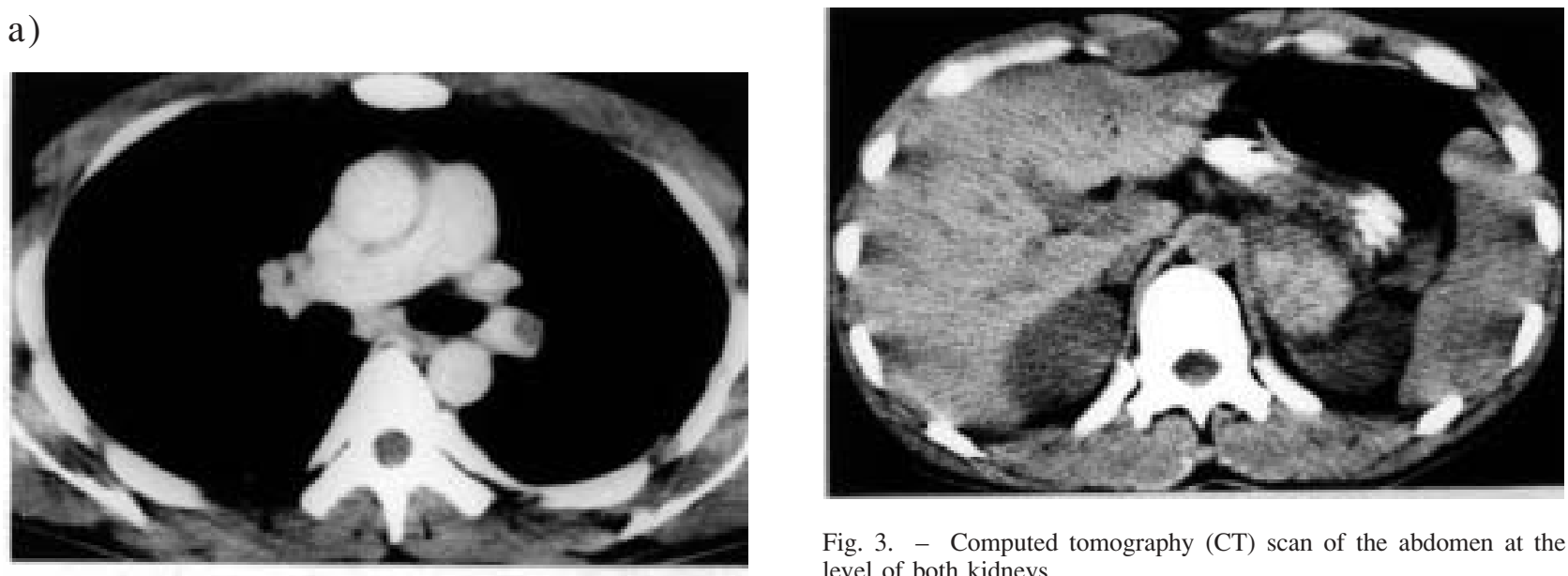

Fig. 3. - Computed tomography (CT) scan of the abdomen at the level of both kidneys.

b)

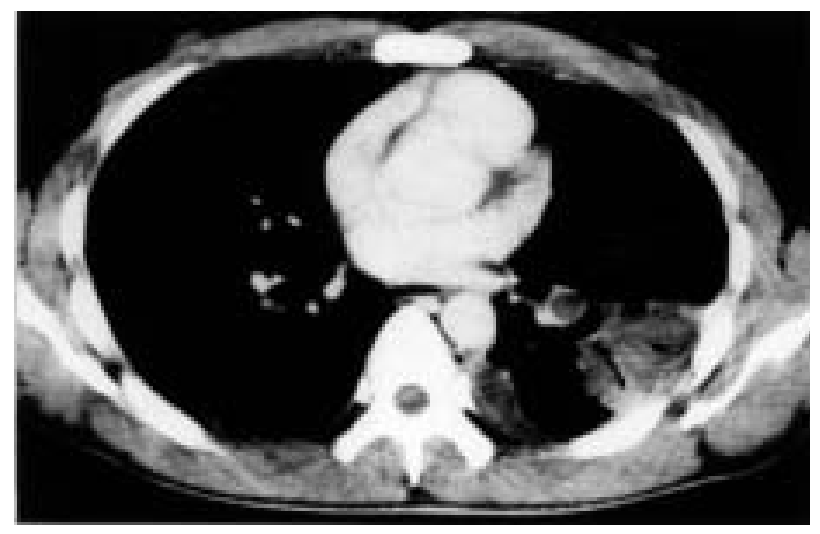

Fig. 2. - a) Computed tomography (CT) scan of the chest at the level of aorticopulmonary window; b) CT section through the lower thorax.

BEFORE TURNING THE PAGE: INTERPRET THE CHEST RADIOGRAPHS AND THE CT SCANS AND SUGGEST THE DIAGNOSIS. 


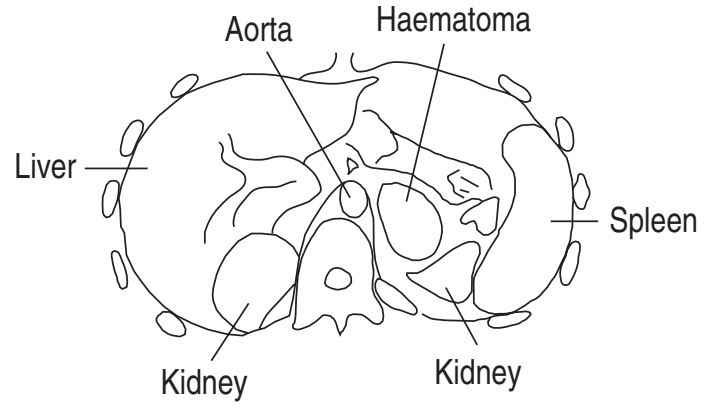

Fig. 4. - Diagrammatic representation of figure 3, showing a highdensity mass in the left suprarenal area suggestive of haematoma.

\section{Interpretation of the chest radiographs and CT}

The first chest radiograph (fig. 1a) reveals right lower lobe opacification with pleural effusion, which resolves almost completely on the follow-up radiograph 5 days later (fig. 1b), whilst left lower lobe consolidation and pleural effusion had developed.

The sequential CT scans of the chest (fig. 2a and b) show an intraluminal filling defect within the left descending pulmonary artery, indicating the presence of an embolus. An additional finding in figure $2 b$ is a wedgeshaped, pleural-based consolidation in the left lower lobe.

The CT section through both kidneys (fig. 3) demonstrates a high-density mass in the left suprarenal area, suggestive of left adrenal haematoma (see the diagramatic representation in figure 4).

Abdominal sonography showed a well-defined, relatively hyperechoic mass about $4 \times 4 \mathrm{~cm}$ in size in the left suprarenal area. Echo-guided aspiration of this mass yielded darkish, noncoagulable blood compatible with recent haemorrhage.

Lung perfusion scan showed wedge-shaped perfusion defects in the entire left lower lobe and posterobasal part of the right lower lobe. A $99 \mathrm{~m}$ Tc-labelled phlebography revealed stasis of flow in the right femoral vein, with prominent collateral circulation. The patient's serum was positive for the anticardiolipin antibody and the lupus anticoagulants; other coagulation profiles were negative. No evidence of other autoimmune diseases or vasculitis was found.

\section{DIAGNOSIS: "Primary antiphospholipid syndrome with pulmonary infarction, pulmonary haemorrhage and left adrenal haemorrhage"}

Thrombolytic therapy with streptokinase was administered and the patient's symptoms gradually improved. Intravenous infusion of heparin was subsequently given for 2 weeks, followed by oral anticoagulation therapy with warfarin. Follow-up CT scan of the chest and abdomen 4 months later disclosed resolution of the filling defect in the left descending pulmonary artery and consolidation in the left lower lobe. The size and density of the left adrenal lesion was also decreased.

\section{Discussion}

This case is presented here in order to alert chest physicians to the association of rapidly changing pulmonary infiltrates with pulmonary thromboembolism (PTE) and pulmonary haemorrhage, and the clinical presentation suggestive of an underlying hypercoagulable state in such a young patient.

The antiphospholipid syndrome (APS) has recently emerged as a major component of collagen/vascular disorders [1-3]. It is a thrombophilic disorder manifested by arterial and/or venous thrombosis, recurrent foetal losses and thrombocytopenia in the presence of antiphospholipid (aPL) antibodies, i.e. lupus anticoagulants (LA) and anticardiolipin (aCL) antibodies [3, 4]. The APS can be divided into primary and secondary types. Primary APS is much more common and occurs in patients who harbour no other underlying disease. Secondary APS is associated with lupus or other autoimmune disorders, malignancy, infection, inflammation, or prolonged use of certain drugs [5]. The antibodies may persist for many years, even lifelong. There is a $2: 1$ female/male sex preponderance compared to the 9:1 ratio in classic systemic lupus erythematosus (SLE) [4]. Vessels of all sizes may be involved [4]. Clinical features of APS are variable, including migraine, visual disturbances, dysarthria, deep vein thombosis (DVT), recurrent miscarriage in mild types and myocardial infarction, major stroke and shock in the serious form ("catastrophic" APS) [1, 4].

Reported pulmonary manifestations of this syndrome include: thromboembolism; pulmonary infarction; diffuse alveolar bleeding with rapidly changing pulmonary infiltrates; pulmonary hypertension; and acute respiratory distress syndrome $[2,5]$. PTE with haemorrhage, without infarction, may appear as an area of groundglass opacification or airspace consolidation on CT scan that is indistinguishable from pneumonia or oedema [6].The diagnosis of a pulmonary infarction based solely on the presence of a peripheral, wedge-shaped lesion is unreliable. The presence of a vascular sign, referring to a thickened vessel leading to the apex of the opacity, increases the likelihood that the lesion represents an infarct [6]. Postembolic alveolar haemorrhage without infarction usually resolves within a week; however, healing of pulmonary infarction typically occurs over 3-5 weeks. In contrast to pneumonic consolidation, which gradually becomes more heterogeneous in attenuation as it resolves, an infarct decreases in size from the periphery whilst maintaining its original shape.

Adrenal haemorrhage is a rare condition, which may be caused by infection, malignancy, thrombocytopenia and anticoagualation therapy. APS-related hypercoagulability may manifest itself as adrenal haemorrhage, which is usually precipitated by anticoagulant therapy, but it may also occur spontaneously [4, 7]. It may be silent and insidious in onset, or acute, occurring after vigorous exercise or during stress, such as infection. In most patients, the adrenal infarction and haemorrhage seem to be secondary to increase in adrenal venous pressure after thromboembolism, as suggested by RAO et al. [8]. It should be considered as one of the differential diagnoses in any patient presenting with adrenal insufficiency or the catastrophic APS. Differentiation from other abdominal emergencies associated with APS, such as mesenteric, splenic, or hepatic infarction is necessary.

Differential diagnosis in APS with pulmonary infiltrates may include diffuse alveolar bleeding secondary 
to various types of vasculitis, particularly Goodpasture's syndrome and Wegener's granulomatosis [5].

Recognition of the association of PTE and adrenal haemorrhage with APS may increase the likelihood of early diagnosis, treatment, and survival in this disorder [9]. However, there is no consensus about the duration and extent of prophylactic antithrombotic treatment. Longterm therapy, in which the international normalized ratio is maintained at or above 3 , is advisable in these patients [3]. The therapeutic role of corticosteroids in primary APS is still controvertial [10].

Keywords: Adrenal haemorrhage, antiphispholipid antibody syndrome, pulmonary thromboembolism.

\section{References}

1. Asherson RA, Cervera R. The antiphospholipid syndrome: a syndrome in evolution. Ann Rheum Dis 1992; 51: $147-150$.

2. Asherson RA, Cervera R. Review: antiphospholipid antibodies and the lung. J Rheumatol 1995; 22: 62-66.
3. Khamashta MA, Cuadrado MJ, Mujic F, Taub NA, Hunt $\mathrm{B}$, Hughes GRV. The management of thrombosis in the antiphospholipid-antibody syndrome. N Engl J Med 1995; 332: 993-997.

4. Bick RL, Baker WF. Antiphospholipid and thrombosis syndrome. Semin Thromb Hemost 1994; 20: 3-15.

5. Hillerdal G, Hagg A, Licke G, Wegenius G, Scheibenpflug L. Intra-alveolar haemorrhage in the anticardiolipin antibody syndrome. Scand J Rheumatol 1991; 20: 58-62.

6. Greaves SM, Hart EM, Brown K, Young DA, Batra P, Aberle DR. Pulmonary thromboembolism: spectrum of findings on CT. AJR 1995; 165: 1359-1363.

7. McCroskey RD, Phillips A, Mott F, Williams EC. Antiphospholipid antibodies and adrenal hemorrhage. Am J Hematol 1991; 36: 60-62.

8. Rao RH, Vagnucci AH, Amico JA. Bilateral massive adrenal haemorrhage: early recognition and treatment. Ann Intern Med 1989; 110: 227-235.

9. Ruffatti A, De Silvestro G, Ghirardello A, et al. A catastrophic antiphospholipid syndrome: the importance of high levels of warfarin anticoagulation. J Intern Med 1994; 235: 81-83.

10. Lee HC, Laya MB. Lethal pulmonary embolism in a patient with primary antiphospholipid syndrome and a vena caval filter. Chest 1994; 105: 312-313. 